ISSN: 2572-2964

Volume 5, Issue 1, 6 Pages

\title{
Independent elbow flexion and extension by isolated long head of triceps transfer for reconstruction of elbow flexion in old upper brachial plexus palsy
}

\author{
${ }^{1}$ Mohammad Abd El Aziz Ali MD, and ${ }^{2}$ Reda H El-kady MD \\ ${ }^{1}$ Assistant professor of Orthopedic Surgery - Zagazig University \\ ${ }^{2}$ Assistant professor of Orthopedic Surgery - Zagazig University
}

\begin{abstract}
Objective: To evaluate the functional outcome of pedicled functional isolated long head of triceps transfer to biceps tendon in failed primary nerve repair or patients seek treatment after 18 month from injury of upper brachial plexus palsy for elbow flexion. Restoring elbow flexion with preserved elbow extension as total transfer of triceps to biceps tendon sacrifices active extension so in our cases we preserve the medial head which is the main elbow extensor and lateral head of triceps.

Methods : Our study, including only patients who had biceps grade $0-2$ and triceps grade $4-5$ in injury of upper brachial plexus palsy, who underwent anterior transfer of isolated long head of the triceps muscle. The affected left side (10), right side (8) sex ( 12 male ) ( 6 female ), type of accident (obstetric brachial plexus palsy (6) , traumatic brachial plexus palsy (12) strength of elbow flexion, complications and patient satisfaction were investigated in 18 cases.

Results: the age range was from 3 to 52 years, with a mean of 25.7 years. The minimum time between injury and surgery was 18 months (range 18-74 months). All the patients at final follow up said that they were satisfied with the procedure. Evaluation Of Elbow function according to Pothula ( 2017 ) after Independent elbow flexion and extension by isolated long head of triceps transfer for reconstruction of elbow flexion : very good results in 10 cases (55.6\%), good results in 5 cases ( 27.8 ), fair results in 2 cases ( $11.1 \%$ ) and failure results in 1 case ( $5.6 \%$ ). So Satisfactory results of isolated long head of triceps transfer for reconstruction of elbow flexion in upper brachial plexus palsy was in 15 cases ( $83.4 \%$ ). Fair results was in 2 cases who were in the left side injury which were not the dominant side of the cases and the patients were satisfied and also failure results in 1 case who revised surgery and operated with lateral head of triceps to biceps and tensioning and the patient was satisfied. 3 cases had complications (initial hematoma in 2 cases and insufficient tensioning in 1 case).
\end{abstract}

Conclusion: Restoring elbow flexion with preserved elbow extension as total transfer of triceps to biceps tendon sacrifices active extension. Independent elbow flexion and extension by isolated long head of triceps transfer to biceps tendon in old brachial plexus palsy is effective, simple and less technically demanding procedure.

\section{INTRODUCTION:}

Inability to flex the elbow is a major disability. Patients with permanent loss of C5 and C6 have normal wrist and hand function with reduced sensibility to the thumb and index finger. However, they are unable to position their hand in space and forward activity as they cannot flex the elbow. Restoration of elbow flexion in such cases leads to a dramatic improvement in upper limb function. (1) Steindler (1918): proximal advancement of the common flexor origin at the elbow. (2) Bunnell (1951): modification by interpositional graft of fascia lata between the flexors and the bone humerus. (3) Mayer and Green (1954): transposed part of the medial epicondyle to provide fixation, securing it more laterally on the bone humerus. Clark (1946): Transfer of the 
Independent elbow flexion and extension by isolated long head of triceps transfer for reconstruction of elbow flexion in old upper brachial plexus palsy

inferolateral portion of pectoralis major and Brooks and Seddon (1959): incorporation of the long head of biceps to lengthen the tendone. (4) Carroll and Kleinman (1979): transfer the entire pectoralis major across the acromion and anterior capsule so stabilize the shoulder and reinforce the transfer. (5) Tsai et al. (1983): simultaneous transfer of pectoralis major and minor. Schottstaedt, Larsen and Bost (1955): Complete latissimus dorsi transfer and musculocutaneous flap can be used if indicated. (6)

Triceps transfer was described by Bunnell (1951), Carroll (1952) and Carroll and Hill (1970): in upper trunk brachial plexus injury when failure of 1ry nerve surgery or after 18 months of injury as pedicled muscle transfer is required in absence of good latissimus dorsi to transfer or used in transfer around shoulder, So management is directed to restore elbow flexion to enable hand-to-mouth activities.(7)

This procedure was described for patients with polio or arthrogryposis having loss of elbow flexion. In comparison to other types of muscle transfer, the tri-to-biceps transfer is preferred and is less technically demanding, independent elbow flexion and extension especially with long head transfer while the disadvantages which may occur mainly are: Affection of elbow extension in cases of total transfer of triceps which preserved as the medial head of triceps which is the main elbow extensor so flexion contracture to elbow does not occur. (8-9)

\section{Patients and Methods:}

Eighteen patients with failure of 1ry nerve surgery or after 18 months of injury of upper brachial plexus were managed by pedicled muscle transfer is required in absence of good latissimus dorsi to transfer or used in transfer around shoulder underwent for restoration of elbow flexion. The age range of the patients was 3-52 years with a mean of 25 years, all patients had clinical examination prior to surgery for assessment of the following, shoulder abduction and adduction; elbow flexion and extension; wrist dorsiflexion and flexion; finger flexion and extension. Grading was done as per the Medical Research Council (MRC) scale, and any range of movement at the elbow if present was recorded. All patients had a pre-operative electromyoneurography (EMG) study of biceps and triceps. The pre-operative triceps grade was more than or equal to MRC 4 . The preoperative biceps grade was M0 to M2. Assessment of the post-operative restoring of elbow flexion was graded as follows: Pathula (2017). Very good - Active range of motion (ROM) $<120^{\circ} / \mathrm{MRC} 4-5$. Good - Active ROM of $80^{\circ}-120^{\circ} /$ MRC4. Fair - Active ROM of $<80^{\circ} /$ MRC3. Failure - Active ROM of $<80^{\circ}$ and/MRC less than Grade 3.

\section{Surgical Technique: (FIG. 1)}

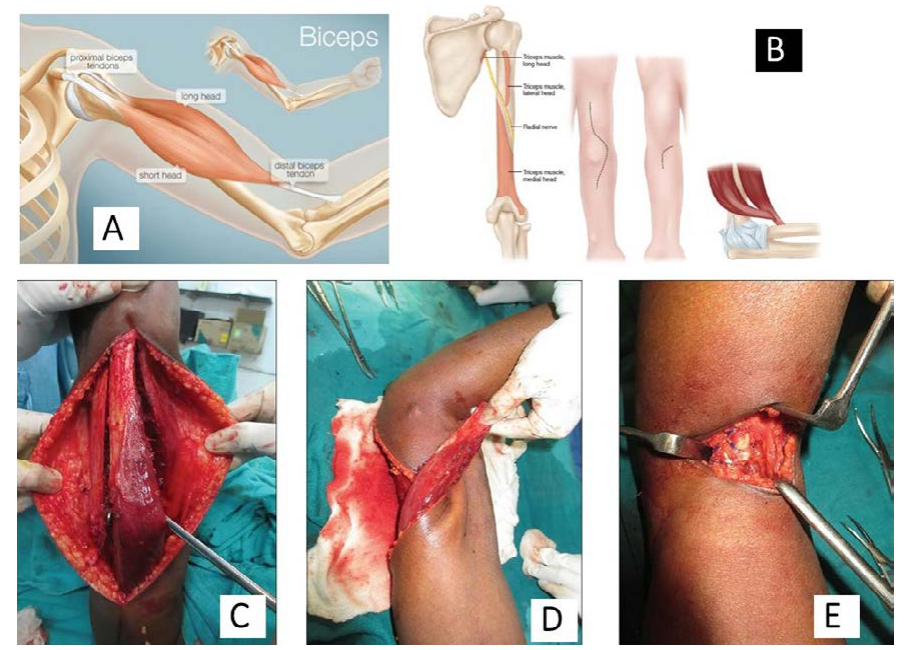

Figure 1: A \& B) Anatomy and surgical incision (15), C, D, and E) intraoperative photos during surgery 
Independent elbow flexion and extension by isolated long head of triceps transfer for reconstruction of elbow flexion in old upper brachial plexus palsy

Considering an isolated long head transfer, an EMG was done to confirm distinct innervation of the long head from the lateral and medial heads of triceps. The patient is placed supine. With the selective transfer of the long head of the triceps, long straight posterior incision is used to identify the vascular pedicle to the long head of the triceps. Dissection of the pedicle leads to the radial nerve branch to the long head of triceps, which is the proximal branch of the radial nerve and is found approximately $10 \mathrm{~cm}$ from the acromion. It is not necessary to dissect out the neurovascular pedicle. Shoulder abduction and elbow flexion make the long head of triceps on maximal stretch, which is then bluntly separated from the medial head. The long head tendon insertion, which is the middle one-third to one-half of the entire triceps insertion, is then released from the olecranon along with an extension of periosteal flap, which is needed for achieving appropriate length. Anterior lazy $\mathrm{S}$ incision from lateral to medial to biceps tendon across elbow crease and the long head of triceps is transferred anteriorly through wide opening by release of lateral septum of the arm and suture end to side of biceps tendon and maximum tensioning done by putting shoulder in $90^{\circ}$ abduction and elbow flexion $90^{\circ}-100^{\circ}$. Haninec and Szeder described transfer of a pedicled long head of triceps for restoring of elbow flexion, and Naidu and colleagues subsequently reported a case report of a patient treated with this procedure. The major advantage of this technique compared with a complete triceps to biceps transfer is that independent active elbow extension can be obtained as the medial head of triceps which is the main elbow extensor. Preoperative electromyography (EMG) of the long head of the triceps demonstrated independent activity separate from the lateral and medial heads. Post-operative (EMG) showed dissociation of elbow flexion from extension. Post-operative follow-up EMG showing disassociation of electrical potentials between transferred head of triceps and the spared two heads triceps. Native triceps is electrically silent when the transferred head of triceps is firing during elbow flexion. Post-operative follow-up with EMG showing the disassociation between the transferred head of triceps and the spared two heads triceps.

\section{RESULTS:}

The mean delay from injury to transfer was 24 months (range 18 to 72 months) and mean follow-up was 36 months (range 24 to 42 months). Most have a good range of active elbow flexion with a mean of $130^{\circ}$ (range 90 to $140^{\circ}$ ); 17 cases were able to bring the hand to the mouth. Power was satisfactory, with ability to lift an average of $3 \mathrm{~kg}$ with the hand when the elbow was at $90^{\circ}$ of flexion (range I to $5 \mathrm{~kg}$ ). All reported considerable functional improvement; most, adequate function is confirmed by the work record. Elbow Flexion MRC (Grades) : in 5 cases elbow flexion grade 5 ( $27.7 \%)$, in 10 cases grade $4(0.55 \%)$ and in 3 cases were grade $3(0.16 \%$ ) 2 of them were in the left side. In right sided case with grade 3 we do tensioning of the tendone transfer and augmented by lateral head transfer to the biceps and sparing medial head of triceps. Power Elbow Flexion at 90 degree ( $1-5 \mathrm{Kg}$ ) : 3 cases can held less than $2 \mathrm{Kg}(16.6 \%), 8$ cases can lift $3 \mathrm{Kg}(44.4 \%), 5$ cases can held from $3-5 \mathrm{Kg}(27.7 \%), 2$ cases can lift more than $5 \mathrm{Kg}(11.1 \%)$. Active range of motion ( ROM ) of elbow flexion post op : Very good ( ROM ) $<120^{\circ}$ in 10 cases $(55.5 \%)$,

Range of motion: (figures: case 2, case 4)
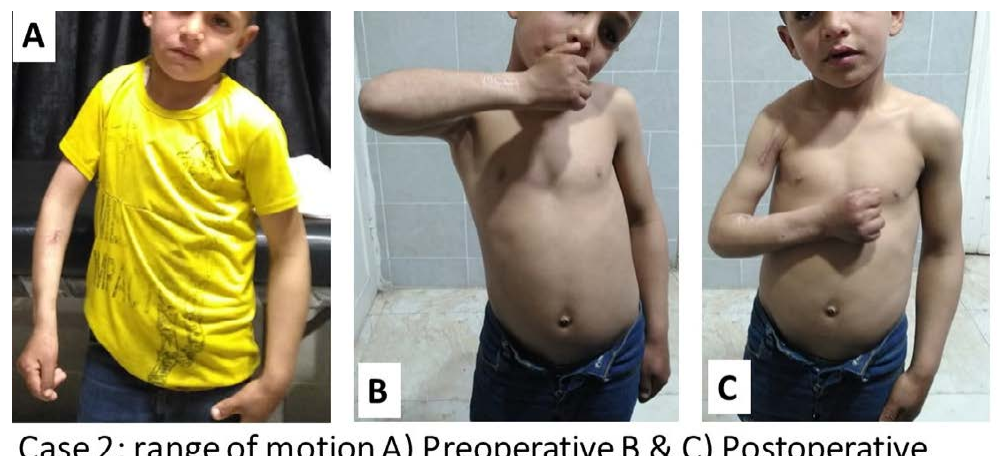

Case 2: range of motion A) Preoperative B \& C) Postoperative 


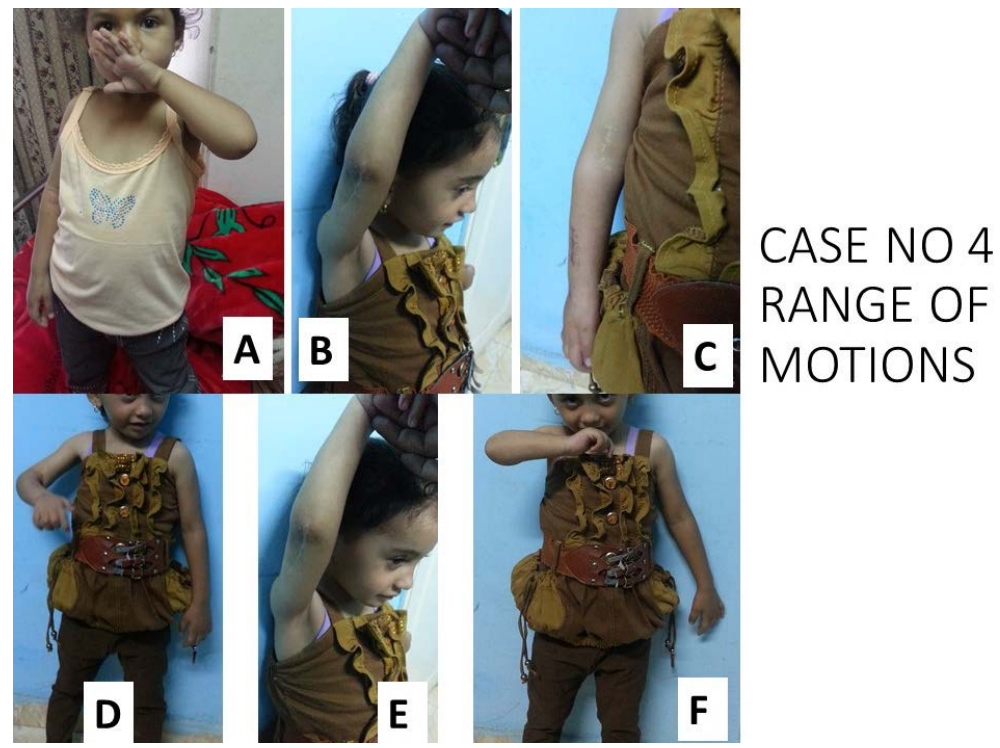

Good (ROM) from $80^{\circ}-120^{\circ}$ in 5 cases ( $27.7 \%$ ), Fair ( ROM ) $80^{\circ}$ in 3 cases $(0.16 \%$ ).

Hand to mouth activity: 3 cases cannot reach hand to mouth (16.6\%), 15 cases can do Hand to mouth activity (83.33\%). Active elbow extension: MRC in 12 cases grade 4 ( $66 \%$ ) and 5 cases grade 3 ( $34 \%$ ). Evaluation Of Elbow function according to Pothula ( 2017 ) after Independent elbow flexion and extension by isolated long head of triceps transfer for reconstruction of elbow flexion in failed primary nerve repair or patients seek treatment after 18 month from injury of upper brachial plexus palsy : very good results in 10 cases (55.6 $\%$ ), good results in 5 cases ( 27.8 ), fair results in 2 cases ( $11.1 \%$ ) and failure results in 1 case ( $5.6 \%$ ). So Satisfactory results of isolated long head of triceps transfer for reconstruction of elbow flexion in upper brachial plexus palsy were satisfied in 15 cases ( $83.4 \%$ ) and fair results was in 2 cases who were in the left side injury which were not the dominant side of the cases and the patients were satisfied and failure results in 1 case who underwent revision surgery operated with lateral head of triceps to biceps and tensioning and the patient were satisfied at final follow up.

\section{Discussion}

In our study, clinical information was most relevant to assessment of a satisfactory results include the overall range of motion, the extensor loss, the ability to bring the hand to the mouth, and the sustained power of the transfer expressed as the load (hand held) which can be supported with the elbow at $90^{\circ}$ of flexion. When we recommend to reconstruct elbow flexion, we should be based on the following considerations, shoulder stability, mechanical advantage of the transferred muscle, work capability of the muscle groups available , musculotendinous excursion, prospect of significant functional gain , range of elbow joint motion, the work capability and mechanical advantage of the muscle transferred, the ideal transfer will follow the route of the biceps muscle, and have a similar excursion , loss of function as a result of transfer and cosmetic. In our study we found that long head of triceps transfer for reconstruction of elbow flexion in upper brachial plexus palsy which originated from infraglenoid tubercle and both head short and long of biceps from supraglenoid and coracoid process of scapula were the best for the transfer that follow these consideration. The work capacity of the different muscles has been studied by Von Lanz and Wachsmuth (1959), who found a significant potential benefit with pectoralis major and triceps transfers. Holtman et al. (1975) have demonstrated the mechanical superiority of pectoralis major and triceps transfers over the Steindler transfers.(10) The disadvantage of a 
Independent elbow flexion and extension by isolated long head of triceps transfer for reconstruction of elbow flexion in old upper brachial plexus palsy

pectoralis major transfer is the destabilizing result on a flail shoulder, unless pectoralis major is transferred (Carroll and Kleinman 1979), and the appearance is unsatisfactory. Patients with a C5/6 palsy have partial denervation of pectoralis major (that part innervated by the lateral pectoral nerve). However, if pectoralis major power is normal, an effective transfer may be present.(11) Latissimus dorsi transfer provides good power but, like the pectoralis transfer, is technically difficult, have a destabilizing effect on the shoulder and is less cosmetically acceptable. Latissimus dorsi has a normal excursion of approximately $11 \mathrm{~cm}$ compared with a biceps tendon range of 5 to $6 \mathrm{~cm}$. The advantage of the triceps transfer is that power is adequate, more than $3 \mathrm{~kg}$ at 90, in both our series and others and is technically straightforward and cosmetically acceptable. The major disadvantage is loss of active extension; this is managed by isolated long head of triceps transfer for reconstruction of elbow flexion in upper brachial plexus palsy and sparing medial head of triceps. Triceps function is critical for upper limb weight bearing such as rising from a chair, and using a crutch or wheel chair; for upper limb activities which demand the ability to press down firmly such as cutting a slice of bread; and for the use of the hand above shoulder height overhead activities. Careful pre-operative counselling on the advantages and disadvantages of the transfer are essential; patients should appreciate that elbow flexion will be regained although there will be reduced activity to press firmly down on hand-held objects. Hoang (1989): had 7 patients who had triceps transfer after an old brachial plexus injury. All patients regained a useful functional improvement with a good range of powerful elbow flexion; 5 patients could manage to bring their hand to their mouth.

Follow-up was 24 months (range 7 to 39 months).(7) All have a good range of active elbow flexion with a mean of $123^{\circ}$ (range $90^{\circ}$ to $140^{\circ}$ ). 5 of 7 cases could bring the hand to the mouth. Some loss of passive extension was found in most cases; the mean loss was $13^{\circ}$ (range 0 to $23^{\circ}$ ). Power was satisfactory, with ability to hold an average of $3.3 \mathrm{~kg}$ with the hand when the elbow was at $90^{\circ}$ of flexion (range 1 to $5 \mathrm{~kg}$ ). The only female patient in the series and has the shortest follow-up, unable to support $2 \mathrm{~kg}$ or more. (7) Fabiano (2010): 10 patients were male; the age range was from 24 to 49 years, and a mean of 33.7 years. The minimum time between injury and surgery was 21 months (range 21-74 months). The left side was affected in 8 cases, and the right in 3. Good results were obtained in 10 patients, who had elbow flexion strength of grade 3 (2cases) and grade 4 (8 cases), but 1 case unfavorably with grade 2 strength. 2 cases had complications (initial compartment syndrome and insufficient tensioning).(11) Loeffler ( 2016): triceps to biceps: total or isolated long head transfer fair power, less technically demanding procedure and independent elbow flexion and extension with long head transfer. Total triceps transfer sacrifices active elbow extension and severe elbow flexion contractures may develop in long term. Total triceps transfer: 5/7 M4 hand to mouth, active flexion arc $123^{\circ}$, and mean $3.3 \mathrm{~kg}$ flexion strength. Long head triceps transfer: 2/2 M4 elbow flexion with preserved M3 elbow extension.(13) Pathula ( 2017 ) : Upper brachial plexus injury in adults causes loss of elbow flexion; when the primary nerve surgery has failed or the patient comes for treatment after 12 months of injury and pedicled muscle transfers are required. One of the heads of triceps muscle to restore the elbow flexion in such cases. In addition to regain of elbow flexion, extension of elbow following surgery is retained. 10 patients suffering from upper brachial plexus injuries underwent transfer of one head of triceps to biceps tendon between December 2011 and August 2015. The recovery of elbow flexion was Grade 5 in 1, Grade 4 in 4 and Grade 3 in 2; only three of the ten cases had no functionally useful elbow flexion. 7 of 10 cases had an adequate elbow extension following the procedure.(14)

In conclusion : our study is simpler and cosmetically more satisfactory. Tendon excursion and muscle power are adequate and the range of flexion is generally satisfactory definite indications for triceps transfer to restore elbow flexion in cases of old upper root plexus palsy. Although pectoralis major and latissimus dorsi transfers may be more powerful than triceps.

Contraindications: Poor passive elbow flexion and presence of lower extremity conditions that require use of upper extremity assistive devices, such as crutches is a relative contraindication. 
Independent elbow flexion and extension by isolated long head of triceps transfer for reconstruction of elbow flexion in old upper brachial plexus palsy

\section{REFERENCES}

Rostoucher P, Alnot JY, Touam C, Oberlin C.: Tendon transfers to restore elbow flexion after traumatic paralysis of the brachial plexus in adults. Int Orthop.1998;22(4):255-62.

Steindler A. : Tendon transplantation in the upper extremity. Am J Surg 1939;XLIV: 260-71.

Bunnell S. : Restoring flexion to the paralytic elbow. J Bone Joint Surg Am 1951;33(3):566-90. Available at: http://jbjs.org/content/33/3/566.abstract.

Brooks DM, Seddon HJ.: Pectoral transplantation for paralysis of the flexors of the elbow; a new technique. J Bone Joint Surg Br 1959;41-B: 36-43.

Chong, J. K., \& Tsai, T. M. (1985). : Restoration of elbow flexion by pectoralis major and pectoralis minor transfer. Plastic and Reconstructive Surgery, 76(6), 982. doi: 10.1097/00006534-198512000-00078

Rühmann O, Schmolke S, Gossé F, Wirth CJ.: Transposition of local muscles to restore elbow flexion in brachial plexus palsy. Injury. 2002;33(7):597-609

Hoang PH, Mills C, Burke FD.: Triceps to biceps transfer for established brachial plexus palsy. J Bone Joint Surg Br 1989;71(2):268-71.

Drake, R. L., Vogl, W., Mitchell, A. W. M., \& Gray, H.: Grays anatomy for students. Philadelphia, PA: Elsevier (2020).

Ann Abarr and Jane Bear : Biomechanics of the elbow, biomechanics of joint part 2 basic biomechanics of the musculoskeletal system. Margreta Nordun third eddetion chapter (14) 2001.

Herring, J.: Tachdjians pediatric orthopaedics from the Texas Scottish Rite hospital for children (2014).

Fabiano Inácio de Souza1, Mateus Saito, Luiz Koiti Kimura,: muscle transfer from triceps to biceps in patients with chronic injury of the upper trunk of the brachial plexus Rev Bras Ortop. 2010;45(4):409-12 .

Haninec P, Szeder V. : Reconstruction of elbow flexion by transposition of pedicled long head of triceps brachii muscle. Acta Chir Plast 1999;41(3):82-6. Available at http://www.ncbi.nlm.nih.gov/pubmed/ 10641328. Accessed March 5, 2016.

Loeffler, B. J., \& Lewis, D. R.: Restoration of Elbow Flexion. Hand Clinics, 32(3), 311-321. doi: 10.1016/j. hcl.2016.03.002.

Pothula Durga Prasada Rao, Rayidi Venkata Koteswara Rao, R. Srikanth : Triceps to biceps transfer for restoration of elbow flexionfollowing upper brachial plexus injury Indian Journal of Plastic Surgery Volume 50 Issue 1 January - April 2017.

Drake, R.L., Vogl, w. 2020. Gray anatomy for students, Philadelphia, PA, Elservier.

Citation: Mohammad Abd El Aziz Ali MD, and Reda H El-kady MD, "Independent elbow flexion and extension by isolated long head of triceps transfer for reconstruction of elbow flexion in old upper brachial plexus palsy". American Research Journal of Orthopedics and Traumatology. 2020; 4(1): 1-6.

Copyright (c) 2020 Mohammad Abd El Aziz Ali MD, and Reda H El-kady MD, This is an open access article distributed under the Creative Commons Attribution License, which permits unrestricted use, distribution, and reproduction in any medium, provided the original work is properly cited. 